CLINICAL STUDY

\title{
The association of cardiac ventricular hypertrophy with congenital hyperinsulinism
}

Indraneel Banerjee ${ }^{1,2}$, Bindu Avatapalle ${ }^{1}$, Anjali Petkar ${ }^{1}$, Mars Skae ${ }^{1}$, Raja Padidela ${ }^{1}$, Sarah Ehtisham ${ }^{1}$, Leena Patel $^{1}$, Lindsey Rigby ${ }^{1}$, Sarah Flanagan ${ }^{3}$, Sian Ellard ${ }^{3}$, Caroline Jones ${ }^{4}$, Giovanna Ciotti ${ }^{4}$, Nilima Malaiya $^{4}$ and Peter E Clayton ${ }^{1,2}$

${ }^{1}$ Department of Paediatric Endocrinology, Royal Manchester Children's Hospital, Oxford Road, Manchester M13 9WL, UK, ${ }^{2}$ Manchester Academic Health Sciences Centre, University of Manchester, Manchester, UK, ${ }^{3}$ Peninsula Medical School, Institute of Biomedical and Clinical Science, University of Exeter, Exeter EX2 5DW, UK and ${ }^{4}$ Department of Paediatric Cardiology, Royal Manchester Children's Hospital, Manchester M13 9WL, UK

(Correspondence should be addressed to I Banerjee at Department of Paediatric Endocrinology, Royal Manchester Children's Hospital; Email: indi.banerjee@cmft.nhs.uk)

\begin{abstract}
Objective: Ventricular hypertrophy (VH) has been observed in children with congenital hyperinsulinism (CHI), a condition of hypoglycaemia characterised by dysregulated insulin secretion, but the prevalence is not known.

Patients and methods: Cardiac assessment was performed in children $(n=49)$ with $\mathrm{CHI}$ at diagnosis and follow-up. Two dimensional and Doppler echocardiography studies were used to assess cardiac structures, while M-mode study was used to measure left ventricular (LV) dimensions, subsequently converted to $\mathrm{Z}$ scores. Where possible, LV hypertrophy was confirmed by LV mass index $\left(\mathrm{g} / \mathrm{m}^{2.7}\right)$ $>95$ th centile.

Results: Cardiac structural lesions were found in 14 (28\%) children. At initial echocardiography, VH was present in $31(65 \%)$ children with median (range) LV posterior wall dimension in diastole $Z$ scores of $+1.6(-2.4$ to +5.8$)$ and interventricular septal wall dimension in end diastole $Z$ scores of +1.9 $(-1.7$ to +17.2$)$. At follow-up echocardiography, performed after an interval of 178 (45-390) days, $\mathrm{VH}$ persisted in $16(33 \%)$ children. In regression analysis, the presence of $\mathrm{VH}$ (odds ratio $(95 \%$ confidence intervals) $1.1(1.0-1.2), P=0.04)$ at initial echocardiography was correlated with maximum glucose requirement at diagnosis, indicating that severity of $\mathrm{CHI}$ at presentation may play a role in the pathogenesis of $\mathrm{VH}$.

Conclusions: A significant proportion of children with $\mathrm{CHI}$ have cardiac structural lesions. A majority also have $\mathrm{VH}$, which may be associated with the severity of $\mathrm{CHI}$ at diagnosis. $\mathrm{VH}$ may persist in some children, which requires careful long-term cardiac review.
\end{abstract}

European Journal of Endocrinology 167 619-624

\section{Introduction}

Congenital hyperinsulinism (CHI) is a rare disorder characterised by hypoglycaemia but is one of the most common causes of persistent hypoglycaemia in infancy and childhood (1). In CHI, secretion of insulin is dysregulated in relation to the level of blood glucose. A significant proportion of children with $\mathrm{CHI}$ is due to genetic defects, most commonly in genes coding for the ATP-sensitive $\mathrm{K}+$ channels $\left(\mathrm{K}_{\text {АTP }}\right.$ channel genes, $A B C C 8$ and KCNJ11) present in the $\beta$ cells of the pancreas (2).

The clinical management of $\mathrm{CHI}$ involves correction of hypoglycaemia by supplemental carbohydrate, medical therapies such as diazoxide and octreotide and pancreatic surgery, the latter depending on the severity, presence of focal lesions and response to medical treatment. Such treatment decisions are usefully aided by rapid $\mathrm{K}_{\text {ATP }}$ channel gene mutation analysis (3). In the initial management of $\mathrm{CHI}$, children often receive large volumes of dextrose solutions to maintain euglycaemia. This may lead to inadvertent fluid overload and pulmonary oedema, unless fluid volumes are restricted by increasing the concentration of dextrose (4). Despite efforts to restrict fluid intake, fluid volume overload leading to features of heart failure is commonly encountered in the clinical management of children with CHI, suggesting the possibility of cardiac dysfunction in CHI.

In addition to volume overload, it is possible that children with CHI may have cardiac muscle hypertrophy, which could contribute to myocardial dysfunction. There are anecdotal reports of reversible hypertrophic cardiomyopathy in association with CHI $(5,6)$, although underlying mechanisms leading to cardiac muscle changes are unknown. It is also well recognised 
that infants born to diabetic mothers have cardiomegaly, even after controlling for birth weight $(7,8)$. The presence of left ventricular hypertrophy (LVH) can be potentially life threatening and has been associated with the risk of sudden death, often due to arrhythmias (9, 10). Therefore, patients with $\mathrm{CHI}$ may be at risk of significant cardiac morbidity, particularly in longterm follow-up.

Despite the possibility of cardiac muscle involvement in CHI leading to myocardial dysfunction, echocardiography to assess cardiac muscle thickness and function is not a routine part of clinical monitoring in several clinical reviews $(4,11,12,13,14)$. In our centre, echocardiography is performed routinely as part of clinical management of children with $\mathrm{CHI}$, although the timing of echocardiography is not standardised. We have now reviewed our echocardiography analyses in $\mathrm{CHI}$ patients with reference to the presence of structural cardiac lesions and ventricular hypertrophy (VH).

\section{Objectives}

To review echocardiography results to identify the prevalence and persistence of $\mathrm{VH}$ in children with $\mathrm{CHI}$.

\section{Patients and methods}

We reviewed echocardiography results at diagnosis and follow-up in a cohort of children with $\mathrm{CHI}$ in a single centre. Case notes were reviewed in a retrospective audit for all children with $\mathrm{CHI}$ undergoing echocardiography over the previous 5 years $(n=49)$. Children with complex congenital heart disease requiring cardiac surgery were included in this review but ventricular parameters to assess $\mathrm{VH}$ were not analysed in this group. The assessment of $\mathrm{VH}$ was limited to those without complex congenital heart defects requiring cardiac surgery $(n=48)$.

The diagnosis of $\mathrm{CHI}$ was made on biochemical assessment, in association with an increased glucose requirement and absence of ketogenesis (11). Severity of CHI was assessed by maximum glucose requirement expressed as $\mathrm{mg} / \mathrm{kg}$ per day. Genotyping for mutations in $\mathrm{K}_{\text {ATP }}$ channel (ABCC8 and KCNJ11), HNF4A, GLUD1 and GCK genes was assessed following diagnosis of CHI. Oral diazoxide was used as first-line treatment, with octreotide used in those who were non-responsive to diazoxide. PET-CT scan using 18 fluoroDopa was performed to diagnose focal CHI. All children were followed in paediatric endocrinology clinics at variable intervals depending on glycaemic stability at clinical assessment.

Children with $\mathrm{CHI}$ underwent cardiac assessment by one of two paediatric cardiologists ( $\mathrm{G} \mathrm{C}$ and $\mathrm{N} \mathrm{M}$ ) at the earliest opportunity after diagnosis of $\mathrm{CHI}$ and where possible before commencing diazoxide. The presence of a patent ductus arteriosus (PDA) was not considered as pathologic unless accompanied by other structural lesions or flow abnormalities or persistence at followup echocardiography. The presence of a patent foramen ovale was not considered as a structural lesion. Atrial septal defect (ASD) was considered pathologic if the child required further follow-up assessment.

$\mathrm{VH}$ was determined as left ventricular (LV), right ventricular (RV) or biventricular (BV), depending on the ventricle affected. The diagnosis of LVH was made by a paediatric cardiologist if $\mathrm{LV}$ dimensions exceeded the upper end of the normal range expected for the child's age and weight and were recorded in the case notes. Quantitative parameters for $\mathrm{LVH}$, i.e. $\mathrm{LV}$ posterior wall dimension in end diastole (LVPWd) and interventricular septal wall dimension in end diastole (IVSd) measurements, were obtained where possible $(n=33)$. These parameters have been widely used in M-mode echocardiography in children and are repeatable and reproducible (15). LV parameters were recorded in centimetres and converted to $\mathrm{Z}$ scores using reference standards from a cohort of healthy children from Europe (16). In addition to LV parameters, where possible, LV mass index (LVMI, g/m ${ }^{2.7}$ ) was measured (17) and values $>95$ th centiles (18) were considered to be confirmatory of LVH $(n=33)$. In regression analysis testing, $\mathrm{VH}$ was considered as an ordinal variable when testing for association with variables related to the type and severity of $\mathrm{CHI}$.

The presence of RV hypertrophy (RVH) was based on subjective assessment without the use of quantitative parameters. Electrocardiogram (ECG) voltage criteria were used to corroborate the presence of RVH in those in whom a diagnosis of RVH was made. ECG was not used for the diagnosis of LVH. Fractional shortening (FS) and ejection fraction (EF) were measured to quantify systolic function of the left ventricle. Diastolic dysfunction was assessed informally and recorded only if significantly abnormal.

\section{Results}

A total of 49 children (37 males) with CHI underwent cardiac assessment and echocardiography after diagnosis. The median (range) age at presentation of hypoglycaemia was 1 (1-300) days. Eight children presented late (range 60-300 days). The first echocardiography was performed 26 (1-89) days after diagnosis of $\mathrm{CHI}$, with $95 \%$ of children undergoing scanning between 18 and 33 days. A second follow-up echocardiography was performed at varying intervals of 178 (45-390) days after initial echocardiography.

Forty-three children underwent genotyping with 22 (51\%) children having $\mathrm{K}_{\mathrm{ATP}}$ channel gene mutations. Of those with mutations, 15 were heterozygous and seven were either homozygous or compound heterozygous. One child had a mutation in $H A D H$, while no mutations were identified in ABCC8, KCNJ11, HNF4A, 


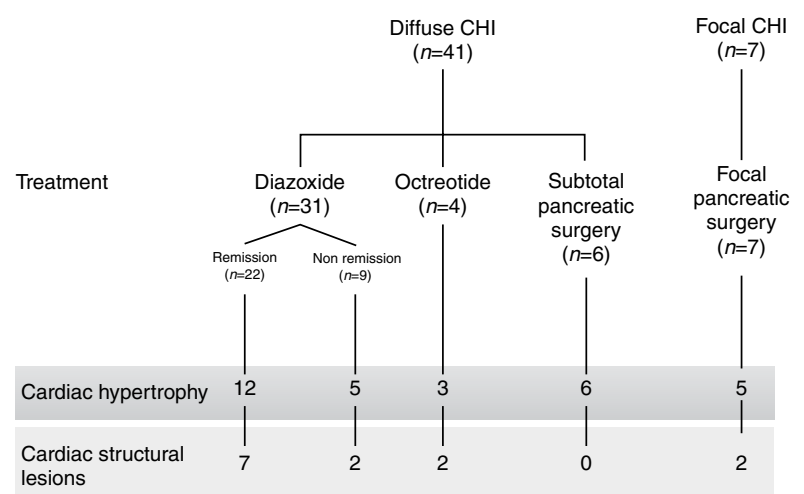

Figure 1 Treatment outcomes and overall M-mode echocardiography findings in children with diffuse and focal $\mathrm{CHI}$ in this cohort $(n=48)$.

GLUD1 or GCK genes in 19 (45\%) children. A flow diagram outlining $\mathrm{CHI}$ treatment outcomes and overall M-mode echocardiography findings is given in Fig. 1.

\section{Cardiac structural lesions}

ASD, PDA, pulmonary stenosis (PS), ventricular septal defect (VSD), aortic stenosis (AS), mitral valve prolapse, double outlet right ventricle (DORV) and malposed great arteries were found in $14(28 \%)$ children. ASD was the commonest abnormality and was present in isolation in four children and in association with PS and VSD in another two children. Mild AS was present in one child with normal aortic arch and normal valvular structure, with no evidence of significant LV outflow tract obstruction (initial aortic tract outflow velocity $2 \mathrm{~m} / \mathrm{s}$ ). Systolic function was satisfactory with EF $76 \%$. In follow-up after 4 weeks, there was no evidence of AS (velocity $1.5 \mathrm{~m} / \mathrm{s}$ ) and no surgical intervention was required. This child had LVH, but the cause was unlikely to be secondary to the relatively mild AS. In two children with PS, the severity was noted as mild and moderate with peak velocity $<3$ and $3 \mathrm{~m} / \mathrm{s}$ respectively. There was no ECG evidence of RVH. In follow-up assessment, surgical intervention was not required for either of the two children. One child with DORV and malposed great arteries had complex cardiac surgery and was therefore excluded from analysis of VH. None of the other children in this cohort required cardiac surgery. In those with cardiac structural lesions $(n=13)$, eight children had LVH, one child had biventricular hypertrophy (BVH) and four children did not have any evidence of $\mathrm{VH}$.

\section{VH at initial and follow-up echocardiography}

VH was present at initial echocardiography in 31 $(65 \%)$ children in this cohort, with LVH in $26(55 \%)$, RVH in two $(4 \%)$ and BVH in three $(6 \%)$ children. VH was observed in $70 \%$ of those undergoing initial echocardiography within 33 days, compared with $63 \%$ of those undergoing initial echocardiography later $(P=0.6$ for difference). At follow-up echocardiography, persistence of $\mathrm{VH}$ was noted in 16 (33\%) children.

At initial echocardiography, LVMI (83.9 (47.1-150.9)) $\mathrm{g} / \mathrm{m}^{2.7}$ values confirmed the presence of LVH in 21 out of 33 children, where such measurements were obtainable. In one child who was born with intrauterine growth retardation with a birth weight of $1.74 \mathrm{~kg}$, LVMI was $<95$ th centile, but other parameters suggested LVH at the age of 2 weeks. In another child of normal weight, LVMI was $>95$ th centile, but other parameters did not suggest LVH. This child had follow-up scans, which were reported as normal.

The median (range) LVPWd at initial echocardiography was $0.4(0.2-0.9) \mathrm{cm}$ with derived $Z$ scores of $+1.6(-2.4$ to +5.8 ) (Fig. 2). In comparison, LVPWd at follow-up echocardiography for 11 patients in whom $\mathrm{LV}$ parameters were available was $0.5(0.3-0.7) \mathrm{cm}$ with $\mathrm{Z}$ scores of $+1.3(-0.4$ to +3.6$)$. There was no significant reduction in LVPWd Z scores between initial and follow-up echocardiography $(P=0.9)$, suggesting the persistence of $\mathrm{LVH}$ at a later date. The median (range) IVSd at initial echocardiography was widely variable at $+0.5(+0.2$ to +5.0$) \mathrm{cm}$ with $\mathrm{Z}$ scores of $+1.9(-1.7$ to +17.2$)$. At follow-up echocardiography, IVSd was $0.5(0.4-1.0) \mathrm{cm}$ with $Z$ scores of +2.1 $(+0.3$ to +7.7$)$ (Fig. 2), but the difference from initial echocardiography was not significant $(P=0.6)$. The presence of cardiac structural lesions was not correlated with the presence of $\mathrm{VH}(P=0.6)$ at initial echocardiography. Similarly, there was no difference in LVPWd $\mathrm{Z}$ scores between those with and without cardiac structural lesions $(P=0.9)$.

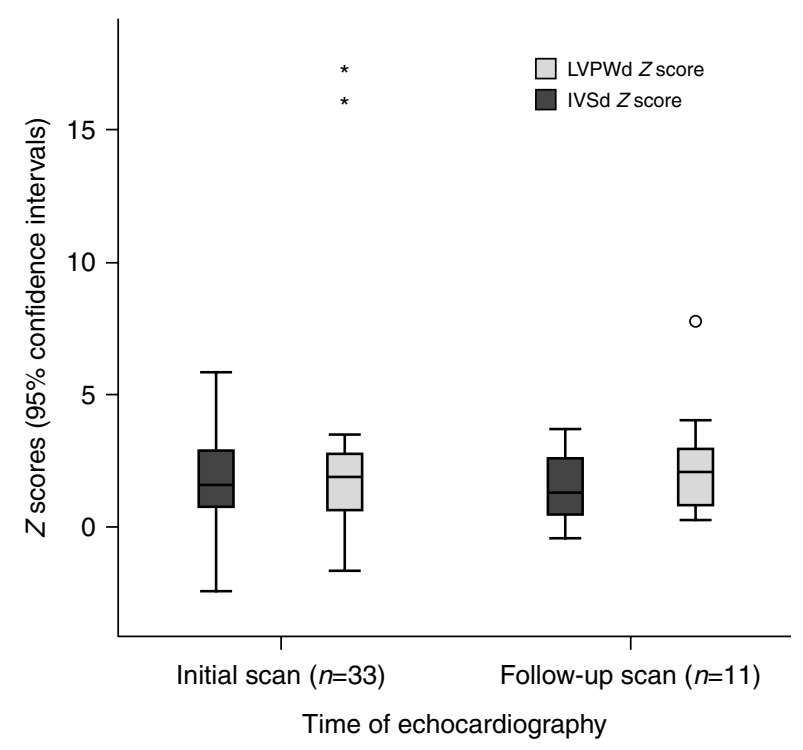

Figure 2 Box plots (median, 95\% confidence intervals and outliers) of $Z$ scores for LVPWd and IVSd parameters at initial and follow-up echocardiography in children with $\mathrm{CHI}$. The asterisks and the open circle indicate outliers. 
There was no correlation of FS with LVPWd $(P=0.4)$ or IVSd $(P=0.6) Z$ scores at initial echocardiography. FS at initial echocardiography was $38(23-56) \%$ and that at follow-up echocardiography was similar at 43 $(27-50) \%(P=0.4$ for difference). EF was normal in all children (74 (51-88) \%, normal values $>50 \%$ in this age group) and did not correlate with LVPWd $(P=0.8)$ and IVSd $(P=0.7)$. No child had significant diastolic dysfunction; therefore, measurement of diastolic function markers was not formally recorded. All children were stable in clinical follow-up and none required long-term cardiac medication in the form of diuretics, ACE inhibitors, digoxin or inotrope support. One child (patient 29) had supraventricular tachycardia at diagnosis, which resolved later. However, this child did not have evidence of cardiac structural lesions or VH.

\section{VH in relation to factors influencing CHI outcomes}

The presence of $\mathrm{VH}$ was assessed in relation to factors characterising $\mathrm{CHI}$ disease severity and outcomes. $\mathrm{K}_{\mathrm{ATP}}$ channel gene mutations, severity of hypoglycaemia (maximum glucose requirement) at presentation and achievement of spontaneous remission of CHI were not associated with a higher probability of $\mathrm{VH}$, either at initial or at follow-up echocardiography, when tested independently. For those treated with diazoxide, initial LVPWd $(1.9(-0.9$ to +5.8$)$ vs $1.2(-2.4$ to +2.9$)$, $P=0.03)$ but not IVSd $(P=0.5) \mathrm{Z}$ scores were relatively higher in those responsive to diazoxide than in those who were unresponsive to diazoxide and therefore treated by octreotide or surgery. However, at follow-up echocardiography, LVPWd $(P=0.7)$ and IVSd $(P=0.3)$ $\mathrm{Z}$ scores were similar between those treated with or without diazoxide.

VH at initial echocardiography was similar in children with focal $\mathrm{CHI}$ as in those with diffuse $\mathrm{CHI}$ (5 $(71 \%)$ vs $26(63 \%), P=0.6$ for difference), in keeping with initial LVPWd $(P=0.08)$ and IVSd $Z$ scores $(P=0.5)$. At follow-up echocardiography, no child with focal CHI treated with lesionectomy had VH. In contrast, $16(39 \%)$ children with diffuse $\mathrm{CHI}$ had persistent $\mathrm{VH}(P=0.04)$.

We investigated the possible correlation of $\mathrm{VH}$ at initial echocardiography with birth weight and gender in addition to the variables tested independently as earlier in a regression equation. In backward Cox regression $(-2 \log$ likelihood 124.0, $P=0.04)$, maximum glucose requirement correlated with the presence of initial VH (odds ratio (95\% confidence intervals, CI) $1.1(1.0-1.2), P=0.04)$ when age at echocardiography was used as a time variable. In backward linear regression, children achieving remission appeared to have a greater degree of LVH at initial echocardiography with LVPWd Z score coefficient (CI) of 1.7 (0.4-3.0), $P=0.009$. In contrast, maximum glucose requirement was positively correlated with IVSd Z score
(0.4 (0.1-0.7), $P=0.006)$, while birth weight was also positively correlated $(1.2(0.05-2.5), P=0.04)$, as expected. However, at follow-up echocardiography, no variable was correlated with the persistence of $\mathrm{VH}$ $(-2 \log$ likelihood 31.4, $P=0.9)$.

\section{Discussion}

In this study, we observed that a significant proportion (28\%) of children with $\mathrm{CHI}$ had cardiac structural lesions. A majority (65\%) of children with $\mathrm{CHI}$ had evidence of $\mathrm{VH}$ at initial echocardiography, by subjective assessment, LVHs and Z score analysis of commonly used M-mode echocardiography parameters. The presence of $\mathrm{VH}$ was independent of body weight, gender and the presence of $\mathrm{K}_{\text {АTP }}$ channel gene mutations. However, maximum glucose requirement, indicating severity of CHI at diagnosis, was correlated with VH. Follow-up echocardiography showed a reduction in the presence of $\mathrm{VH}$, with persistence in $33 \%$ of children with CHI.

The association of $\mathrm{VH}$ and increased incidence of cardiac structural lesions has not been described in children with CHI. The association of LVH and fatal arrhythmias is well recognised in adults; using the same principles, it would be important to recognise the presence of $\mathrm{VH}$ in patients with $\mathrm{CHI}$ with a view to intervene with medical therapy, if necessary, to prevent cardiac dysfunction and the potential development of life-threatening arrhythmias.

Although the review uncovers an important area in the long-term management of patients with $\mathrm{CHI}$, we accept that our analysis suffers from inherent weaknesses in study design. This is a retrospective analysis where initial and follow-up echocardiograms were not suitably standardised. As a consequence, there has been significant variation in the intervals to initial and follow-up echocardiography. However, Z score analysis of M-mode echocardiogram dimensions and time variable corrections in regression analyses suggest significant association of $\mathrm{VH}$ in $\mathrm{CHI}$ patients despite temporal non-standardisation.

Measurements of outflow tract obstruction have not been analysed in the study as records were obtainable only in a small proportion of children. However, in those with PS, the degree of obstruction was well characterised. In one child with AS and LVH, it was unlikely that LVH was a consequence of the structural lesion and was therefore more likely due to CHI. Another weakness of this study is the lack of echocardiography assessment for diastolic dysfunction to characterise LVH from a functional perspective. However, this being a pilot observational study, the need for detailed Doppler trans-mitral velocity studies was not anticipated. It is expected that future study protocols will incorporate more rigorous investigation modalities.

The finding of LVH in children with $\mathrm{CHI}$ is in keeping with anecdotal observations and case reports $(5,6)$ and 
is similar to that seen in infants of diabetic mothers $(7,8)$. Some children with $\mathrm{CHI}$ are relatively macrosomic at birth and hence may have a greater cardiac muscle mass. In our study, we controlled for the effects of birth weight, gender and age at presentation by conversion of $\mathrm{LV}$ parameters to $\mathrm{Z}$ scores and noted the presence of $\mathrm{VH}$ even after adjusting for body size.

Maximum glucose requirement, corresponding to output of insulin and hence the severity of CHI, was correlated with $\mathrm{VH}$ at initial echocardiography, when adjusting for other variables. This indicates that the severity of CHI may influence the degree and persistence of VH. However, in our cohort, we observed that children who achieved remission of $\mathrm{CHI}$ had greater LV parameters, indicating LVH at initial echocardiography. While it is known that remission of CHI is not correlated with markers of severity (3), the association of remission with LVH in this cohort may suggest that initial CHI severity may play a greater part in the pathogenesis of $\mathrm{VH}$. In children with focal CHI, the amelioration of $\mathrm{VH}$ following lesionectomy supports this notion. In contrast, the correlation of cardiac structural lesions and persistent VH may suggest different mechanisms of pathogenesis responsible for the incidence and persistence of $\mathrm{VH}$.

All children in this cohort had been initially treated with diazoxide as first-line treatment for CHI. It is possible that $\mathrm{VH}$ was secondary to the use of diazoxide, while acting on $\mathrm{K}_{\text {АтP }}$ channels also present in the myocardium. However, $\mathrm{VH}$ was also present in some children before commencing diazoxide, which indicates that the influence of diazoxide as a causative factor for $\mathrm{VH}$ is unlikely. Further, in rat models, diazoxide appears to reduce $\mathrm{VH}$, suggesting that $\mathrm{VH}$ observed in children with CHI is not causally associated with diazoxide (19).

We were not able to determine the cause of cardiac structural lesions in this retrospective study. For a more definitive assessment of factors influencing $\mathrm{VH}$, a larger cohort should be examined for longitudinal trends in $\mathrm{LV}$ parameters with growth, $\mathrm{CHI}$ treatment and achievement of remission. Although in our cohort systolic function was normal and no child required long-term medication for cardiac dysfunction, diastolic function was not formally recorded. Therefore, the natural history and long-term outcome of $\mathrm{VH}$ will need further evaluation.

It is now known that $\mathrm{K}_{\text {ATP }}$ channels that are present in $\beta$ cells in the pancreas are also present in rat atria and ventricles with differential responses to therapeutic agents (20). It is possible that $\mathrm{K}_{\text {ATP }}$ channel abnormalities may be a common factor underlying $\mathrm{CHI}$ and $\mathrm{VH}$ in children, but as yet, there is no evidence to support this notion. Further, abnormal $\mathrm{K}_{\text {ATP }}$ channel function is unlikely to explain $\mathrm{VH}$ in those without $\mathrm{K}_{\mathrm{ATP}}$ channel gene mutations, in children responsive to diazoxide treatment and in those achieving spontaneous remission, i.e. in conditions with adequate pancreatic $\mathrm{K}_{\mathrm{ATP}}$ channel activity.

\section{Conclusions}

In this cohort of patients with CHI, echocardiography detected the presence of cardiac structural lesions in a significant proportion $(28 \%)$ of children. A majority $(65 \%)$ of children with $\mathrm{CHI}$ had VH, mostly of the left ventricle, which was confirmed by analysis of $\mathrm{LV}$ echocardiography parameters. It is possible that factors influencing the severity of $\mathrm{CHI}$ may be involved in the pathogenesis of $\mathrm{VH}$. VH may persist at follow-up echocardiography, implying the need to investigate long-term cardiac outcomes in all children with CHI.

We would recommend that echocardiography should be performed to exclude structural cardiac lesions and quantify LVH. Repeat echocardiography should be performed, even after improvement of hypoglycaemia, until VH is no longer present.

\section{Declaration of interest}

The authors declare that there is no conflict of interest that could be perceived as prejudicing the impartiality of the research reported.

\section{Funding}

This research did not receive any specific grant from any funding agency in the public, commercial or not-for-profit sector.

\section{Author contribution statement}

All authors in this manuscript have contributed substantially to the manuscript, either in design, data collection or analysis, and drafting of the article and have provided final approval before submission of the manuscript.

\section{References}

1 Kapoor RR, James C \& Hussain K. Advances in the diagnosis and management of hyperinsulinemic hypoglycemia. Nature Clinical Practice. Endocrinology \& Metabolism 20095 101-112. (doi:10.1038/ncpendmet1046)

2 James C, Kapoor RR, Ismail D \& Hussain K. The genetic basis of congenital hyperinsulinism. Journal of Medical Genetics $20094 \mathbf{6}$ 289-299. (doi:10.1136/jmg.2008.064337)

3 Banerjee I, Skae M, Flanagan SE, Rigby L, Patel L, Didi M, Blair J. Ehtisham S, Ellard S, Cosgrove KE, Dunne MJ \& Clayton PE. The contribution of rapid $\mathrm{K}_{\mathrm{ATP}}$ channel gene mutation analysis to the clinical management of children with congenital hyperinsulinism. European Journal of Endocrinology 2011 164 733-740. (doi:10.1530/EJE-10-1136)

4 Aynsley-Green A, Hussain K, Hall J, Saudubray JM, Nihoul-Fekete C, De Lonlay-Debeney P, Brunelle F, Otonkoski T, Thornton P \& Lindley KJ. Practical management of hyperinsulinism in infancy. Archives of Disease in Childhood. Fetal and Neonatal Edition $2000 \mathbf{8 2}$ F98-F107. (doi:10.1136/fn.82.2.F98)

5 Harris JP, Ricker AT, Gray RS, Steed RD \& Gutai JJ. Reversible hypertrophic cardiomyopathy associated with nesidioblastosis. Journal of Pediatrics 1992120 272-275. (doi:10.1016/S00223476(05)80442-1)

6 Massin MM, Van Elmbt G \& Soyeur D. Reversible hypertrophic cardiomyopathy in congenital hyperinsulinism. Acta Cardiologica $199954359-361$. 
7 Mormile R, De Michele M, Squarcia U \& Quaini F. Hypertrophic cardiomyopathy in neonates of diabetic mothers: indirect evidence for a model of apoptotic reversibility by survivin? International Journal of Cardiology 2011 146 244-245. (doi:10.1016/j.ijcard.2010.10.058)

8 Russell NE, Holloway P, Quinn S, Foley M, Kelehan P \& McAuliffe FM. Cardiomyopathy and cardiomegaly in stillborn infants of diabetic mothers. Pediatric and Developmental Pathology 200811 10-14. (doi:10.2350/07-05-0277.1)

9 Barbieri A, Bursi F, Mantovani F, Valenti C, Quaglia M, Berti E, Marino M \& Modena MG. Left ventricular hypertrophy reclassification and death: application of the Recommendation of the American Society of Echocardiography/European Association of Echocardiography. European Heart Journal Cardiovascular Imaging 201213 109-117. (doi:10.1093/ejechocard/jer176)

10 Bulbul A, Bolat F, Comert S, Demirin H, Tanik C, Bulbul L, Nuhoglu A \& Dokucu AI. Persistent hyperinsulinemic hypoglycemia with left ventricular hypertrophy and dysrhythmia: a case report. Fetal and Pediatric Pathology 201029 165-171. (doi:10.3109/15513811003777334)

11 Arnoux JB, Verkarre V, Saint-Martin C, Montravers F, Brassier A, Valayannopoulos V, Brunelle F, Fournet JC, Robert JJ, Aigrain Y, Bellanne-Chantelot C \& de Lonlay P. Congenital hyperinsulinism: current trends in diagnosis and therapy. Orphanet Journal of Rare Diseases 20116 63. (doi:10.1186/1750-1172-6-63)

12 De Leon DD \& Stanley CA. Mechanisms of disease: advances in diagnosis and treatment of hyperinsulinism in neonates. Nature Clinical Practice. Endocrinology \& Metabolism $2007 \quad 3$ 57-68. (doi:10.1038/ncpendmet0368)

13 Kapoor RR, Flanagan SE, James C, Shield J, Ellard S \& Hussain K. Hyperinsulinaemic hypoglycaemia. Archives of Disease in Childhood 200994 450-457. (doi:10.1136/adc.2008.148171)

14 Senniappan S, Shanti B, James C \& Hussain K. Hyperinsulinaemic hypoglycaemia: genetic mechanisms, diagnosis and management. Journal of Inherited Metabolic Disease 201235 589-601. (doi:10.1007/s10545-011-9441-2)
15 Geelhoed MJ, Snijders SP, Kleyburg-Linkers VE, Steegers EA, van Osch-Gevers L \& Jaddoe VW. Reliability of echocardiographic measurements of left cardiac structures in healthy children. Cardiology in the Young 200919 494-500. (doi:10.1017/S10 47951109990862)

16 Kampmann C, Wiethoff CM, Wenzel A, Stolz G, Betancor M, Wippermann CF, Huth RG, Habermehl P, Knuf M, Emschermann T \& Stopfkuchen H. Normal values of M mode echocardiographic measurements of more than healthy infants and children in central Europe. Heart 200083 667-672. (doi:10.1136/heart.83. 6.667)

17 Foster BJ, Mackie AS, Mitsnefes M, Ali H, Mamber S \& Colan SD. A novel method of expressing left ventricular mass relative to body size in children. Circulation 2008117 2769-2775. (doi:10.1161/ CIRCULATIONAHA.107.741157)

18 Khoury PR, Mitsnefes M, Daniels SR \& Kimball TR. Age-specific reference intervals for indexed left ventricular mass in children. Journal of the American Society of Echocardiography 200922 709-714. (doi:10.1016/j.echo.2009.03.003)

19 Xia Y, Rajapurohitam V, Cook MA \& Karmazyn M. Inhibition of phenylephrine induced hypertrophy in rat neonatal cardiomyocytes by the mitochondrial $\mathrm{K}_{\mathrm{ATP}}$ channel opener diazoxide. Journal of Molecular and Cellular Cardiology 200437 1063-1067. (doi:10.1016/j.yjmcc.2004.07.002)

20 Zhang HX, Akrouh A, Kurata HT, Remedi MS, Lawton JS \& Nichols CG. HMR 1098 is not an SUR isotype specific inhibitor of heterologous or sarcolemmal $\mathrm{K}_{\mathrm{ATP}}$ channels. Journal of Molecular and Cellular Cardiology 201150 552-560. (doi:10.1016/j.yjmcc. 2010.12.011)

Received 10 June 2012

Accepted 15 August 2012 